\title{
A INTERFACE ENTRE A DOENÇA PERIODONTAL E A REABILITAÇÃO COM PRÓTESE PARCIAL REMOVIIVEL: UMA REVISÃO DE LITERATURA
}

\author{
THE INTERFACE BETWEEN PERIODONTAL DISEASE AND \\ REHABILITATION WITH REMOVABLE PARTIAL DENTURE: A LITERATURE \\ REVIEW
}

\begin{abstract}
Weslley Souza Petyk ${ }^{1 *}$, Maria Luisa Gomes Ferreira¹, Valéria Lima Avelar²
1 Graduando em Odontologia pela Universidade Estadual de Maringá (Maringá/PR, Brasil).

${ }^{2}$ Especialista em Prótese e Reabilitação oral pela UNINGÁ, Mestre em Odontologia pela Universidade Estadual de Londrina e Doutoranda em Odontologia pela Universidade Estadual de Maringá (Maringá/PR, Brasil).
\end{abstract}

*Rua Bragança, n 89, Maringá/PR, Brasil. CEP 87.020-220. E-mail: weslleypetyk@gmail.com

Submetido em: 28/12/2019; Aceito em: 16/06/2020.

\section{RESUMO}

Um fator etiológico da doença periodontal é o biofilme. Pelo seu acúmulo, ele pode desencadear fatores de retenção locais, dentre eles estão as próteses parciais removíveis (PPRs). A função desse aparelho protético é substituir os dentes ausentes em pacientes desdentados parcialmente. A relação entre periodontia e prótese dentária na construção de um plano de tratamento integrado permite a manutenção da função da reabilitação protética, com boa expectativa de duração do tratamento. Esta revisão tem como objetivo analisar os estudos científicos presentes na literatura, que relacionem a prótese parcial removível e a doença periodontal. Foram selecionados artigos nas bases de dados eletrônicas PUBMED e Biblioteca Virtual da Saúde (BVS) no período de 2000 a 2018, nos idiomas português, inglês e espanhol. Foram utilizados termos em inglês indexados no Medical Subject Heading Terms (Mesh Terms -MESH) para o PUBMED e termos em português indexados nos "Descritores em Ciências da Saúde" (DECS) para a plataforma BVS. Os estudos analisados mostraram que a PPR aumenta o risco de desenvolver doenças periodontais, dentre elas: periodontite e gengivite. $\mathrm{O}$ aumento do risco está relacionado à negligência na higienização e ao planejamento indevido da PPR. Sendo assim, a associação de uma terapia periodontal com o controle de biofilme adequado e periódico em interface a um planejamento e adaptação da PPR pode ser uma boa alternativa para se evitar danos ao remanescente dental e ao periodonto.

Palavras-chave: Doenças periodontais. Odontologia. Prótese Dentária.

\section{ABSTRACT}

An etiologic factor of periodontal disease is the dental biofilm. Through its accumulation, it can trigger local retention factors, among them are the removable partial dentures (RPD). The function of this prosthetic device is to replace missing 
teeth in partially toothless patients. The relationship between periodontics and dental prosthesis, in the construction of an integrated treatment plan, allows the maintenance of prosthetic rehabilitation's function with a good duration's expectancy. This review aims to analyze the scientific studies present in the literature which relate the removable partial dentures and the periodontal disease. Some articles were selected in PUBMED electronic databases and Virtual Health Library (BVS) from 2000 to 2018, in the Portuguese, English and Spanish. English terms indexed in the Medical Subject Heading Terms (Mesh) were used for PUBMED; and terms in Portuguese indexed in the "Descriptors in Health Sciences" (DECS) for the BVS platform. The studies demonstrated that RPD increases the risk of developing periodontal diseases, including periodontitis and gingivitis. The increased risk is related to negligence in hygiene and improper planning of RPD. Thus, the association of a periodontal therapy with the control of adequate and periodic plaque in interface to a planning and adaptation of RPD may be a good alternative to avoid damage to the dental and periodontal remnant.

Keywords: Dental Prothesis. Dentistry. Periodontal Disease.

\section{INTRODUÇÃO}

O edentulismo, seja parcial ou total, é considerado um problema social, pois acarreta a diminuição da capacidade funcional, reduz a eficiência mastigatória, além de causar danos estéticos e psicológicos (PERES et al., 2013). A perda dos elementos dentários pode ser elucidada com a reabilitação oral protética, pelas próteses totais, parciais ou implantes (CARDOSO; BUJES, 2010).

A prótese parcial removível (PPR) é uma opção de reabilitação oral nos casos de edentulismo parcial, pois há uma impossibilidade na confecção de próteses fixas ou implantes, principalmente associada às questões financeiras (SILVA; PORTO; BONACHELLA, 2008). As intervenções protéticas com PPRs objetivam melhorar a qualidade de vida do paciente, visto que, além de repor elementos dentários ausentes, solucionam questões estéticas e funcionais (CARDOSO; MACHADO, 2003).

Uma das principais causas de insucesso no uso de PPRs são as doenças periodontais. Tais enfermidades agridem os tecidos de suporte dos dentes, representando-se por alterações de textura e cor gengival, formação de bolsas periodontais, recessões gengivais e, em alguns casos, a perda dentária (NYMAN; LINDHE, 1977).

Vários estudos demonstraram que o biofilme constitui o principal fator etiológico das doenças periodontais, ainda que para a manutenção da saúde bucal seja de extrema relevância o controle do índice de placa (LÖE; THEILADE; JENSEN, 1965; TRISTÄO et al., 1989; ALBANDAR, 2002). O biofilme se caracteriza como um ecossistema microbiano aderido à superfície do dente e o seu acúmulo pode ser favorecido e potencializado por aspectos locais, como o uso de PPRs (BERGMAN, 1987).

Segundo alguns autores, o uso da PPR está associado ao acúmulo de biofilme, principalmente em dentes onde acessórios ou grampos estão 
instalados (GHAMRAWY, 1976; ADDY, 1978; STIPHO, 1978), relacionando o emprego disso ao aparecimento de doenças periodontais (ADDY, 1977; TUOMINEN, 1989; ORR, 1992). Ainda de acordo com Zlatari, Elebi e ValentiPeruzovi (2002), Vacaru et al. (2003) e Sesma et al. (2005), os desenhos da estrutura da PPR, a forma da base da prótese, a construção da prótese e a quantidade de grampos atuam no acúmulo de biofilme, causando uma possível deterioração periodontal.

No entanto, alguns estudos, recomendam aspectos que acreditam minimizar o risco de aparecimento da doença periodontal com o uso de PPRs, como: planejamento, destacando a biomecânica e o desenho da PPR, higienização e controles periódicos ao dentista (ADDY; BATES, 1978; MCHENRY et al., 1992; KAPUR et al., 1994; BERGMAN; HUGOSON; OLSSON, 1995; VANZEVEREN; D'HOORE; BERCY, 2002; ZLATARIC; ELEBI; VALENTIPERUZOVI, 2002; SALZEDAS et al., 2003).

Uma pesquisa na literatura sobre o assunto, buscando os aspectos essenciais do amplo comprometimento da doença periodontal na população e sua iminente relação com o uso de próteses parciais removíveis, é plenamente justificável. Dessa forma, o objetivo do presente estudo foi identificar, na literatura, a relação do tratamento com PPR e o efeito disso na deterioração dos tecidos periodontais.

\section{METODOLOGIA}

Para esta revisão da literatura, realizou-se uma busca avançada nas bases de dados eletrônicas PubMed e Biblioteca Virtual da Saúde (BVS), no período de 2000 a 2018, utilizando-se termos em inglês indexados no Medical Subject Heading Terms (Mesh Terms - MeSH) para o Pubmed e termos em português indexados nos "Descritores em Ciências da Saúde" (DeCs) para a plataforma BVS.

Na plataforma PubMed, a sequência da pesquisa, originada a partir do cruzamento das palavras-chave com o operador booleano AND foi: ("Periodontal Diseases"[Mesh]) AND "Denture, Partial, Removable"[Mesh]. Na busca foram encontrados 716 trabalhos, sendo 228 selecionados para a leitura dos títulos e resumos. A primeira seleção utilizou o filtro para a busca de artigos em idioma português, inglês ou espanhol, entre 2000 e 2018, excluindo dissertações, teses e capítulos de livros.

A pesquisa na plataforma BVS foi gerada com base nos termos: "Periodontal disease" AND "Removable partial denture". A busca recuperou 487 registros, sendo 170 selecionados para a leitura dos títulos e resumos. A primeira seleção utilizou o filtro para a busca de artigos em idioma português, inglês ou espanhol, entre 2000 e 2018, excluindo dissertações, teses e capítulos de livros.

Dentre a literatura obtida, foram lidos os títulos e resumos, sendo selecionados 27 artigos que tratavam do tema proposto para a leitura do texto completo. Dentre eles foram excluídos 14 artigos, sendo que 3 não foram encontrados na íntegra, 4 não tratavam especificamente do tema proposto e 7 eram repetidos. Dessa forma, foram selecionados 13 trabalhos que melhor corresponderam aos objetivos propostos por este estudo (Figura 1). 
Figura 1 - Fluxograma descritor do processo de seleção dos estudos para revisão.

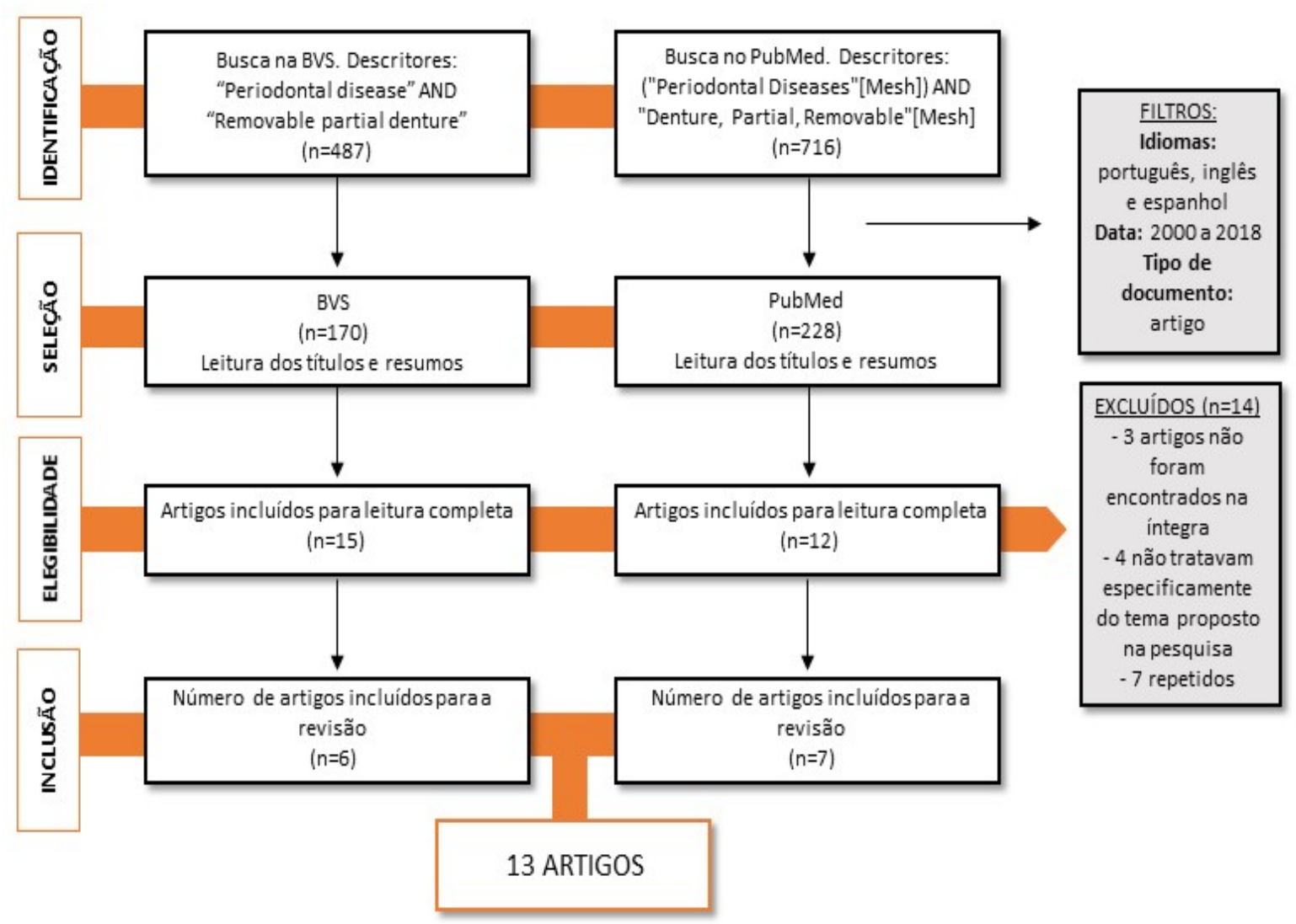

Fonte: os autores.

\section{DESENVOLVIMENTO}

Índice de placa (IP), Profundidade de sondagem (PS), Sangramento à sondagem (SS), Mobilidade dentária (MD) e Recessão gengival (RG)

O uso de prótese parcial removível (PPR) pode ser um fator local de retenção de biofilme, que influencia na mudança da microbiota bucal e pode acarretar problemas periodontais, como gengivite e periodontite. Isso leva ao sangramento à sondagem, aumento na profundidade da sondagem, mobilidade dental e perda óssea (YEUNG et al., 2000). Essas modificações podem ocorrer por falta de uma terapia periodontal após a entrega da PPR e uma deficiente higienização bucal.

Yeung et al. (2000), em um acompanhamento de 5 a 6 anos, com 87 pacientes, relataram que após a instalação da PPR, os sítios dentários que se encontravam em contato com a armação metálica apresentaram um aumento significativo no IP, na PS, no SS e a presença de RG comparado aos dentes que não tinham contato com a prótese. Os pacientes que realizavam visitas periódicas apresentaram, proporcionalmente, um menor acúmulo de biofilme e sangramento gengival.

Zlataric, Elebi e Valenti-Peruzovi (2002) analisaram 205 pacientes que utilizavam PPR por um período de 1 a 10 anos. Ao comparar os dentes pilares e 
não-pilares da prótese para alguns índices de doença periodontal, concluíram que os dentes pilares da PPR demonstraram um maior grau de IP, PS, MD e $R G$, comparado aos dentes não-pilares, indicando ser mais difícil a higienização dos dentes pilares. Contudo, se houver um correto planejamento, com instrução de higiene oral, pode-se diminuir o aparecimento da doença.

Em um trabalho similar realizado por Correia et al. (2018) em 54 pacientes reabilitados com PPR, por um período de 3 anos, eles observaram que os dentes pilares apresentaram os valores de todos os parâmetros periodontais analisados (PS, RG, IP e MD) consideravelmente maior comparado aos dentes não pilares.

Dula et al. (2015) também pesquisaram a influência de PPRs sobre a saúde periodontal de dentes pilares e não pilares. Eles acompanharam 107 pacientes, por um período de 3 meses e se observou que no primeiro mês, apenas o IP apresentou um aumento significativo. Após três meses, houve diferenças significativas entre os dentes do pilar e do não pilar em relação ao SS, PS e IP.

Kern e Wagner (2001) analisaram as mudanças ocasionadas pelo desenho das PPRs em relação às condições periodontais diante de um longo período de uso. Eles analisaram 147 pacientes e o estado periodontal dos dentes pilares e não pilares foram avaliados de imediato e após um período de 10 anos em relação à inserção das próteses. Foi encontrada uma alta taxa de extração de dentes pilares e não pilares, a PS e o SS dos dentes restantes se deterioraram. No entanto, essa deterioração foi maior nos dentes do pilar do que nos dentes não pilares.

Carreiro, Dias e Lopes (2017) avaliaram 22 pacientes antes e depois de 7 anos, com o uso da prótese. Os dados clínicos mostram que houve uma diferença estatisticamente significante para RG e PS entre os três grupos (pilar direto, pilar indireto e dente não pilar). Os valores mais altos foram encontrados nos dentes pilares diretos, em comparação aos pilares indiretos e não pilares. Embora observado um aumento do SS no decurso, não houve diferença estatística entre os três grupos.

Em um estudo semelhante, Amaral et al. (2010), ao estudarem durante um ano 50 pacientes, mostraram que os dentes não envolvidos no desenho da prótese foram os menos afetados para todas as variáveis estudadas (PS e IP). Segundo os autores, isso acontece pois os dentes que recebem os elementos da prótese são mais susceptíveis a acumular maiores quantidades de biofilme, além de prejudicar a ação de autolimpeza realizada pela saliva, língua e bochechas e a própria higiene bucal deficiente do paciente. Verificou-se também, que os valores médios de PS aumentaram após um ano de uso de PPR em todos os grupos (pilar direto, pilar indireto e não pilar), mas que apenas o IP apresentou um aumento considerável. $\mathrm{O}$ estudo indicou que os retentores diretos e indiretos tendem a causar maiores problemas periodontais associado ao uso da PPR, quando comparados com dentes não pilares.

Jorge, Giampaolo e Vergani (2007) acompanharam durante seis meses um estudo clínico e avaliaram o grau de mobilidade de dentes pilares. Não houve alteração significativa na mobilidade dentária durante o período acompanhamento, independentemente do design. Esses resultados, porém, são contrários a alguns estudos que mostram dano ao periodonto. Zlataric, Elebi e Valenti-Peruzovi (2002) e Correia et al. (2018) concluíram que as PPRs afetam 
a mobilidade dos dentes pilares de forma negativa. No entanto, os autores observaram que quando realizado um planejamento correto no desenho da prótese, associado às consultas periódicas e instruções de higienização, é possível prevenir alterações que levam à mobilidade dentária.

Atualmente, vários estudos têm sido executados para verificar o efeito das PPRs na saúde periodontal e, principalmente, no acúmulo de biofilme, sangramento à sondagem, mobilidade dentária, profundidade de sondagem, demonstrando resultados desfavoráveis quando não há orientações sobre técnicas de higiene oral e controle por revisões periódicas. Algumas pesquisas que avaliaram o efeito da PPR na saúde periodontal mostraram que os dentes pilares da prótese tiveram um aumento significativo dos danos periodontais, comparado aos dentes não pilares (KERN; WAGNER, 2001; ZLATARIC; ELEBI; VALENTI-PERUZOVI, 2002; DULA et al., 2015; CORREIA et al., 2018).

Em relação ao aparecimento da mobilidade dentária e ao uso da PPR, os trabalhos não obtiveram um consenso. Entretanto, alguns autores concordam que o planejamento para a execução da prótese é de enorme importância para reduzir as forças nos dentes pilares, o que poderia levar à mobilidade dentária (ZLATARIC; ELEBI; VALENTI-PERUZOVI, 2002; JORGE; GIAMPAOLO; VERGANI, 2007; CORREIA et al., 2018).

A maioria dos estudos sugerem que o uso da PPR está relacionado a uma maior formação de biofilme ao redor dos dentes remanescentes, podendo acarretar problemas periodontais como a inflamação gengival, levando ao sangramento na sondagem, aumento na mobilidade dentária e recessão gengival. Entretanto, vários estudos concluíram que o controle adequado do biofilme pela implementação de rigorosos programas de manutenção e excelentes medidas de higiene bucal e da prótese, podem prevenir problemas periodontais, garantindo a funcionalidade e longevidade da PPR e, portanto, o bem-estar do paciente (YEUNG et al., 2000; KERN; WAGNER, 2001; ZLATARIC; ELEBI; VALENTI-PERUZOVI, 2002; AKALTAN; KAYNAK, 2005; AMARAL et al., 2010; DULA et al., 2015).

\section{O design da PPR e as condições periodontais}

Para a confecção da prótese parcial removível (PPR) é necessário conhecer os materiais odontológicos, procedimentos laboratoriais e os princípios biológicos dos componentes da estrutura. Caso não seja bem planejado e executado, isso pode trazer sérios problemas ao paciente, como 0 comprometimento do periodonto de suporte (SANTOS et al., 2007). As próteses parciais removíveis são constituídas por conectores, dentes artificiais, retentores e sela.

Os retentores são classificados como diretos (que se relacionam com dentes pilares vizinhos ao espaço protético) e indiretos (que são propostos para os dentes pilares situados distantes do espaço protético) (DI FIORI, 1993). Addy e Bates (1978) verificaram que nas superfícies linguais e vestibulares os dentes apresentavam maior acúmulo de biofilme e as PPR inferiores com conectores maiores, do tipo placa lingual, acumularam significativamente mais biofilme do que a barra lingual. $O$ estudo evidencia que quanto maior o tempo de uso da prótese, o acúmulo de biofilme e sangramento gengival são maiores, isso acontece pois a PPR ocasiona o aumento de biofilme no arco oposto à prótese, 
o que diminui o potencial de limpeza dos tecidos moles pela saliva e pelo processo mastigatório, associado à higiene bucal deficiente. Foi constatado pelos autores que o impacto periodontal tende a ser menor na utilização de próteses fixas quando comparadas com as PPRs. No entanto, os efeitos negativos não justificam a contraindicação das PPRs, desde que tenham as suas indicações feitas corretamente, com o objetivo de permitir o bem-estar do paciente.

Zlataric, Elebi e Valenti-Peruzovl (2002) afirmaram que a configuração da PPR possui influência na saúde dos tecidos periodontais. Observou-se que nos pacientes usuários de próteses inferiores, contendo como conector maior a placa lingual, obtiveram valores maiores de profundidade de sondagem (PS) e índice de placa (IP). Akaltan e Kaynak (2005) realizaram um estudo de 30 meses para avaliar os efeitos das PPRs tipo placa lingual e barra lingual na saúde periodontal de 36 pacientes. A descoberta mais notável do estudo foi que, com exceção da recessão gengival $(R G)$, as condições periodontais melhoraram com os dois tipos de PPRs. Ao fim dos 30 meses, o índice de placa (IP) foi maior no grupo da placa lingual e os valores de recessão gengival $(R G)$ e mobilidade dentária (MD) foram maiores no grupo barra lingual. Todescan, Silva e Silva (2003) indicam o preparo de apoios profundos por uma restauração protética, pois assim há distribuição entre os pontos de aplicação da força mastigatória para a região cervical da coroa, diminuindo, dessa forma, o centro de força. Há indicação pelos autores do grampo RPA (apoio mesial, placa proximal distal e grampo circunferencial). Assim, o seu braço de retenção facilitaria a higienização, pois estaria acima da recessão.

Alves e Lima (2001) afirmam que há um maior acúmulo de biofilme, tanto na área conector maior/tecido gengival quanto na área grampo/dente. Por isso, o desenho dos conectores deve respeitar os princípios para facilitar a higienização e prevenir impactação alimentar. Quando a extremidade do conector maior é posicionada na porção central do dente de suporte, tem-se como efeito negativo a retração gengival, a reabsorção óssea e, em alguns casos, a mobilidade dentária. A eficiência do preparo de planos-guia aumenta quando mais de uma superfície do mesmo suporte é preparada, por exemplo, por lingual e por proximal. Essa condição é de extrema importância em dentes periodontalmente tratados, cujos suportes se apresentam com mobilidade aumentada ou com extensa perda de estrutura óssea, sendo necessária a obtenção de estabilização conferida pela PPR (ALI et al., 2001).

\section{Perda dentária relacionada ao uso de PPRs}

Kern e Wagner (2001) analisaram o estado periodontal de 74 pacientes durante 10 anos após a instalação da PPR. Foi identificado que o número de dentes restantes diminuiu de 606 para 482 no acompanhamento de 10 anos, com um número maior de dentes pilares sendo perdidos durante esse tempo $(26,4 \%)$, em comparação com os dentes não-pilares (14,2\%). Os autores concluíram que há uma alta taxa de extração dentária em usuários de PPRs e os dentes que foram extraídos eram suscetíveis a terem sido os mais gravemente afetados pela doença periodontal. Tada et al. (2015) analisaram 1094 dentes pilares, desses, 164 foram perdidos no final do período de observação. A causa da perda dentária devido à doença periodontal foi de $42 \%$. 


\section{CONCLUSÃO}

Com base nesta revisão de literatura, o uso da PPR está relacionado a uma maior formação de biofilme. Se não bem adaptada, ela pode prejudicar os dentes pilares com problemas periodontais, como a inflamação gengival, levando ao sangramento na sondagem, aumento na mobilidade dentária e recessão gengival. Entretanto, vários estudos concluíram que o controle adequado do biofilme pela implementação de rigorosos programas de manutenção, associado a um planejamento e execução da prótese podem evitar problemas periodontais. Outras medidas complementares e que irão promover 0 sucesso das próteses parciais removíveis são a indissociabilidade entre próteseperiodontia-prevenção, ocasionando que a PPR cause pouco ou nenhum efeito deletério ao periodonto.

\section{REFERÊNCIAS}

ADDY, M.; BATES, J. F. Plaque accumulation following the wearing of different types of removable partial dentures. Journal of Oral Rehabilitation, v. 6, p. 111$117,1978$.

ADDY, M.; BATES, J. F. The effect of partial dentures and chlorhexidine gluconate gel an plaque accumulation in the absence of oral hygiene. Journal of Clinical Periodontology, v. 4, p. 41-47, 1977.

AKALTAN, F.; KAYNAK, D. An evaluation of the effects of two distal extension removable partial denture designs on tooth stabilization and periodontal health. Journal of Oral Rehabilitation, v. 32, p. 823-829, 2005.

ALBANDAR, J. M. Global risk factors and risk indicators for periodontal diseases. Periodontology 2000, v. 29, p. 177-206, 2002.

ALI, M. et al. A laboratory investigation of the role of guide planes in the retention of cast cobalt-chromium alloy partial denture frameworks. Journal of Dentistry, v. 29, p. 291-9, 2001.

ALVES, J.; LIMA, N. P. Avaliação periodontal dos pacientes usuários de Prótese Parcial Removível. Revista íbero-americana de Prótese Clínica \& Laboratical, v. 3, p. 137-45, 2001.

AMARAL, B. A. et al. A clinical follow-up study of the periodontal conditions of RPD abutment and non-abutment teeth. Journal of Oral Rehabilitation, v. 37, n. 7, p. 545-552, 2010.

BERGMAN, B. Periodontal reactions related to removable partial dentures: a literature review. Journal of Prosthetic Dentistry, v. 58, p. 454-8, 1987. 
BERGMAN, B.; HUGOSON, A.; OLSSON, C. O. A 25 year longitudinal study of patients treated with removable partial dentures. Journal of Oral Rehabilitation, v. 22, p. 595- 599, 1995.

CARDOSO, M. C. A. F.; BUJES, R. V. A saúde bucal e as funções da mastigação e deglutição nos idosos. Estudos Interdisciplinares sobre o Envelhecimento, v. 15, n. 1, p. 53-67, 2010.

CARDOSO, R.; MACHADO, M. Odontologia, Conhecimento e arte: Dentística, Prótese, ATM, Implantodontia, Cirurgia, Odontogeriatria. São Paulo Artes Médicas, p. 472, 2003.

CARREIRO, A. F. P.; DIAS, K. C.; LOPES, A. L. C. Periodontal Conditions of Abutments and Non-Abutments in Removable Partial Dentures over 7 Years of Use. Journal of Prosthodontics, v. 26, n. 8, p. 644-649, 2017.

CORREIA, A. R. M. et al. Evaluation of the Periodontal Status of Abutment Teeth in Removable Partial Dentures. International Journal of Periodontics \& Restorative Dentistry, v. 38, n. 5, p. 755-760, 2018.

DI FIORI, S. R. Atlas de prótese parcial removível. 4a ed. São Paulo: Pancast Editorial. p. 116-164, 1993.

DULA, L. J. et al. The influence of removable partial dentures on the periodontal health of abutment and non-abutment teeth. European Journal of Dentistry, v. 9, p. 382-6, 2015.

GHAMRAWY, E. E. Quantitative changes in dental plaque formation related to removable partial dentures. Journal of Oral Rehabilitation, v. 3, p. 115-20, 1976.

JORGE, J. H.; GIAMPAOLO, E. T.; VERGANI, C. E. Clinical evaluation of abutment teeth of removable partial denture by means of the Periotest method. Journal of Oral Rehabilitation, v. 34, p. 222-227, 2007.

KAPUR, K. K. et al. A randomized clinical trial of two basic removable partial denture designs. Part I: Comparisons of five year success rates and periodontal health. Journal of Prosthetic Dentistry, v. 72, p. 268-282, 1994.

KERN, M.; WAGNER, B. Periodontal in patients 10 years after insertion of removable partial dentures. Journal of Oral Rehabilitation, v. 28, p. 991-997, 2001.

LÖE, H.; THEILADE, E.; JENSEN, S. B. Experimental gingivitis in man. Journal of Periodontology, v. 36, p. 177-87, 1965.

MCHENRY, K. R. et al. Relationship of removable partial denture use to root caries in an older population. The International Journal of Prosthodontics, v. 5, p. 39-46, 1992. 
NYMAN, S.; LINDHE, J. Considerations on the design of occlusion in prosthetic rehabilitation of patients with advanced periodontal disease. Journal of Clinical Periodontology, v. 4, p. 1-15, 1977.

ORR, S.; LINDEN, G. J.; NEWMAN, H. N. The effect of partial denture connectors on gingival health. Journal of Clinical Periodontology, v. 19, p. 589-94, 1992.

PERES, M. A. et al. Perdas dentárias no Brasil: análise da Pesquisa Nacional de Saúde Bucal 2010. Revista de Saúde Pública, v. 47, p. 78-89, 2013.

SALZEDAS, D. C. et al. Avaliação periodontal de dentes retentores de próteses parciais removíveis. Revista Brasileira de Prótese Clínica e Laboratorial, v. 5, n. 23, p. 52-57, 2003.

SANTOS, M. B. F. et al. Longitudinal study of removable partial dentures and hygiene habits. Ciência Odontológica Brasileira, v. 10, n. 3, p. 38-43, 2007.

SESMA, N. et al. Effect of denture surface glazing on denture plaque formation. Brazilian Dental Journal, v. 16, p. 129-34, 2005.

SILVA, P.; PORTO, V.; BONACHELLA, W. Aspectos periodontais em pacientes usuários de prótese parcial removível. Revista Odonto Ciência, p. 297-301, 2008.

STIPHO, H. D.; MURPHY, W. M.; ADAMS D. Effect of oral prostheses on plaque accumulation. British Dental Journal, v. 145, p. 47-50, 1978.

TADA, S. et al. Impact of periodontal maintenance on tooth survival in patients with removable partial dentures. Journal of Clinical Periodontology, v. 4, p. 246-53, 2015.

TODESCAN, R.; SILVA, E. E.; SILVA, O.J. Atlas de prótese parcial removível. São Paulo: Santos; 2003.

TRISTÄO, G. C. et al. Prothesis: dental plaque control. Revista da Associação Paulista de Cirurgiões Dentistas, v. 43, p. 179-82, 1989.

TUOMINEN, R.; RANTA, K.; PAUNIO, I. Wearing of removable partial dentures in relation to periodontal pockets. Journal of Oral Rehabilitation, v. 16, p. 119126, 1989.

VACARU, R. et al. Periodontal-restorative interrelationships. Oral Health Dental, v. 3, p. 12-5, 2003.

VANZEVEREN, C.; D'HOORE, W.; BERCY, P. Influence of removable partial denture on periodontal indices and microbiological status. Journal of Oral Rehabilitation, v. 29, p. 232-239, 2002. 
YEUNG, A. L. et al. Oral health status of patients 5-6 years after placement of cobalt-chromium removable partial dentures. Journal of Oral Rehabilitation, v. 27, p. 183-189, 2000.

ZLATARIC, D. K.; ELEBI, A.; VALENTI-PERUZOVI, M. The effect of removable partial dentures on periodontal health of abutment and non-abutment teeth. Journal of Periodontology, v. 73, p. 137-144, 2002. 